\title{
Innate and Adaptive Immunity in Inflammatory Bowel Diseases
}

\author{
Sebastian Foersch Maximilian J. Waldner Markus F. Neurath \\ Department of Medicine 1, Friedrich Alexander University, Erlangen, Germany
}

\section{Key Words}

Innate immune system · Adaptive immune system

\begin{abstract}
While barrier function and the effects of the intestinal microbiome have only recently moved into the focus of inflammatory bowel disease research, the role of the innate and the adaptive immune system in these gastrointestinal disorders has extensively been studied. Although still not completely understood, the increasing knowledge about the immune system's contribution to the pathophysiology of inflammatory bowel diseases has led to new diagnostic and therapeutic approaches. This review gives a compact overview on this important topic.

๑) 2013 S. Karger AG, Basel
\end{abstract}

\section{Introduction}

Crohn's disease (CD) and ulcerative colitis (UC) are the two entities classically referred to as inflammatory bowel diseases (IBD) [1]. Both CD and UC are characterized by a chronic and relapsing idiopathic inflammation of the gastrointestinal tract. Moreover, IBD is accompanied by extraintestinal manifestations and an increased risk of cancer development [2]. The main clinical symptoms include diarrhea, abdominal pain and fever, and patients are prone to increased morbidity and reduced quality of life. Although CD and UC partly differ in their clinical presentation and underlying pathophysiology, both entities are characterized by the dysfunction of three major mechanisms: (i) the intestinal barrier, (ii) the innate and the adaptive immune system and their interconnection, and (iii) effects of non-immune cells such as fibroblasts and the endothelium of mucosal vessels. While function and impairment of the intestinal barrier only recently moved in the focus of IBD research, the role of both the adaptive and innate immune system has been studied extensively and will be subject to this concise review.

\section{Innate Immune System}

Unlike any other organ, the gastrointestinal tract faces a unique challenge: while the intestinal mucosa has to provide protection from pathogens such as bacteria, fungi and viruses, it also has to tolerate beneficial commensal microbiota and physiological food-associated antigens. This 'hyporeactive' state has to be tightly regulated and seems to turn into immunologic 'hyperreactivity' in patients with IBD. The first barrier is constituted by a mucus

\section{KARGER}

E-Mail karger@karger.com

www.karger.com/ddi
(C) 2013 S. Karger AG, Basel

0257-2753/13/0314-0317\$38.00/0
Prof. Dr. med. Markus F. Neurath

Department of Medicine 1

Friedrich-Alexander-Universität Erlangen-Nürnberg

Ulmenweg 18, DE-91054 Erlangen (Germany)

E-Mail markus.neurath@uk-erlangen.de 
layer consisting of different antimicrobial agents [3], which are secreted by goblet cells and Paneth cells. Although not the focus of this review, these molecules and cell types seem to substantively contribute to the pathogenesis of IBD [4]. The innate immune system constitutes the first line of active immunologic defense. Various receptors such as Toll-like receptors (TLR), nucleotide oligomerization domain (NOD)-like receptors, C-type lectin receptors (CLR) or retinoic acid-inducible gene (RIG-1)-like receptors (RLR) sense different microbial molecules called pathogen-associated molecular patterns (PAMP). These receptors are located on macrophages, dendritic cells (DCs) and other cells of the lamina propria to initiate an inflammatory response [5]. Experimental models have shown some of these receptors to play an important role in IBD. For example, myeloid differentiation factor 88 (MyD88), a TLR signaling adaptor protein, is indispensable for development of enterocolitis in an IL-10-absent and IL-12/23-p40-dysregulated environment [6]. NF- $\kappa B$ deficiency leads to apoptosis of colonic epithelial cells and consecutive translocation of bacteria into the mucosa. Deficiency of the gene encoding the adaptor protein MyD88 prevents the development of intestinal inflammation, demonstrating that TLR activation by intestinal bacteria is essential for disease pathogenesis an experimental model of colitis [7]. Additionally, polymorphisms in the genes for these receptors have been associated with IBD [8].

Macrophages are an integral part of the innate immune system of the gastrointestinal tract [9]. They clear luminal microbes that manage to overcome the intestinal barrier by phagocytosis. Intestinal macrophages are characterized by the markers F4/80, CD115 and CD14, but lack CD103 expression [10]. It has been shown that under healthy conditions, intestinal macrophages show profound anergy and contribute to tissue homeostasis through downregulation of proinflammatory cytokines $[11,12]$. This attenuation mediates physiologic tolerance. In IBD, on the contrary, macrophages together with circulating monocytes orchestrate an exaggerated immunologic response. This is characterized by an increased expression of IL-12, IL-23 and TNF- $\alpha$ in vitro [13]. Animal models further showed an important role of IL-10, which controls immune regulation and macrophage function [14]. IL-10-deficient mice develop spontaneous colitis by shifting macrophage differentiation to a more proinflammatory subset. This can be rescued by macrophage depletion [15].

Apart from macrophages, DCs are the other key cell type of the innate immune system in the gastrointestinal tract. DCs are located in the lamina propria, especially in the Peyer's patches and also in the mesenteric lymph nodes. Under healthy conditions, luminal antigens are presented to DCs by epithelial cells and microfold cells ( $M$ cells). It has been demonstrated that DCs even have dendrites reaching between epithelial cells directly into the lumen [16]. Upon activation, DCs migrate to the mesenteric lymph nodes to interact with $B$ and $T$ cells and thus provide a link between the innate and the adaptive immune system. Moreover, DCs coordinate T-cell generation and differentiation. Under healthy conditions, they can induce regulatory $\mathrm{T}$ cells, which suppress the function of effector $\mathrm{T}$ cells $[14,17]$. In a proinflammatory setting with high levels of IL- 1 and TNF- $\alpha$, as can be found in patients with IBD, DC trafficking is enhanced and they orchestrate the T-cell response (either Th1, Th2 or Th17) [18]. For example, in CD, increased IL-12 production of DCs leads to Th1-mediated inflammation [16].

\section{Adaptive Immune System}

Apart from the innate immune system, the adaptive immune response and their interconnection play an important role under healthy and pathologic conditions. Cells of the adaptive immune system include $T$ and $B$ cells and their various subtypes, and can be found in the mucosa-associated lymphoid tissues (MALT). The general hallmark of these cells are surface receptors whose structures derive from gene rearrangement. This leads to high specificity and enables an immunologic 'memory' function. Although the epithelial layer and the innate immune system constitute a robust barrier, constant representation of antigens of the commensal flora to the peripheral T-cell pool occurs [19]. Landmark studies by Powrie et al. [20] and others showed that the T-cell repertoire includes specificities that can facilitate inflammatory responses to the microbiota. Transferring this cell type into immunodeficient mice depleted of endogenous regulatory cells resulted in the development of features of IBD.

There are currently three described subsets of effector CD4+ T cells: Th1, Th2, and Th17 cells, all of which are also found in CD or UC, respectively. Differentiation into one of these cell subtypes is driven by cytokines mostly released from cells of the innate immune response. Additionally, interconversion between various subsets has been proposed [21]. Th1 response was originally associated with CD and starts with activation of STAT1 and STAT4 by IL-12 and interferons [22]. The specific transcription factor for the Th1 cells is T-bet, surface markers 
Fig. 1. Cell types, chemokines and pathways involved in the pathogenesis of inflammatory bowel disease.

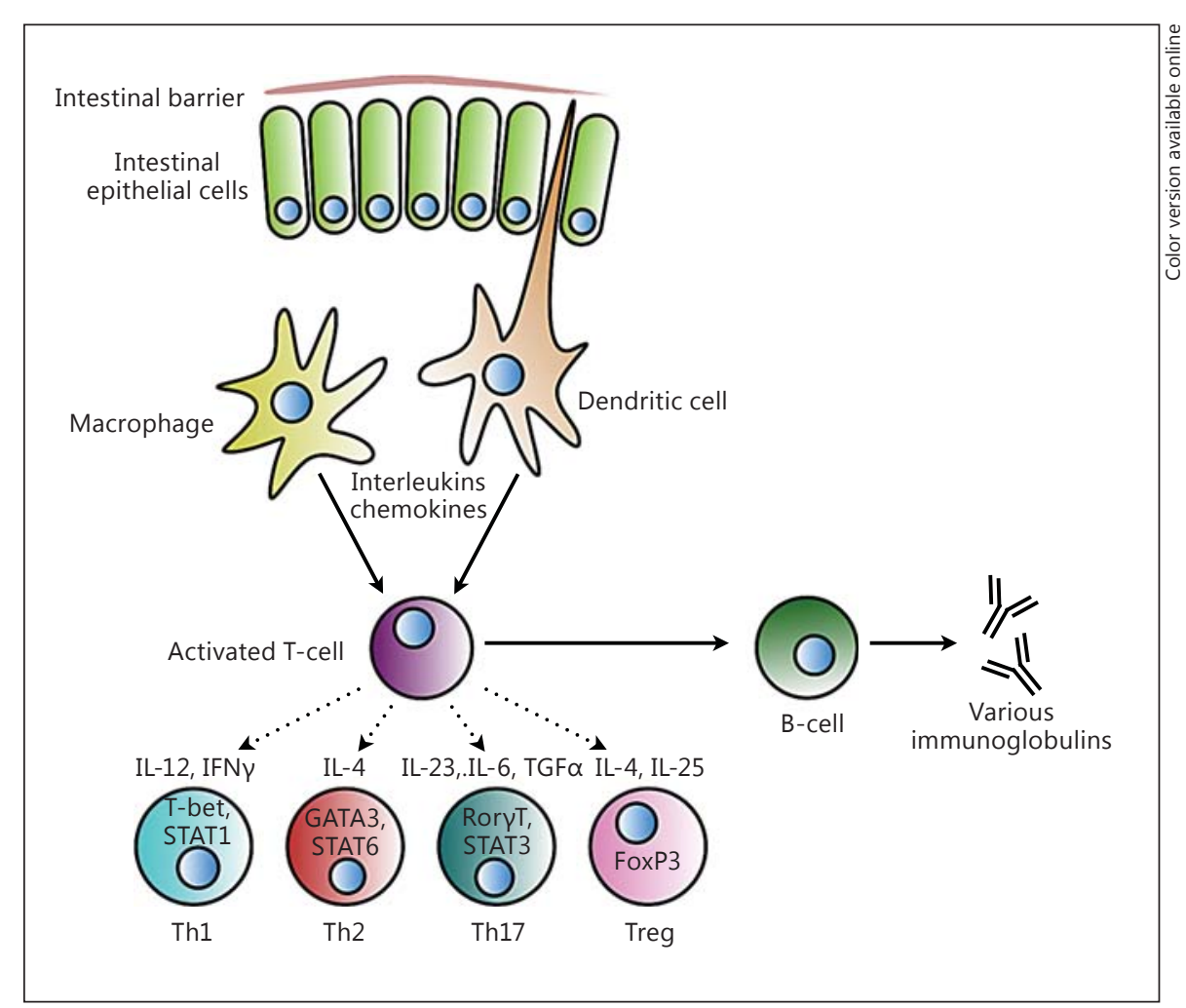

include CCR1 and -5 and CXCR3 [23, 24]. A Th17-cell response is activated for example upon detection of extracellular bacteria and fungi. The differentiation is induced by proinflammatory IL-6, IL-23 and TGF- $\beta$ via STAT3 and ROR $\gamma \mathrm{T}$ [25]. IL-23, which shares the $\mathrm{p} 40$ subunit with IL-12, has been proposed as a key factor in experimental models of CD [26]. IL-17A and IL-17F are Th17 cytokines and involved in neutrophil recruitment and activation [27]. Th17 cells have also been associated with $\mathrm{CD}$. UC, on the other hand, is connected to a modified Th2 response associated with IL- 5 and IL- 13 responses that results in the specific activation of transcription factor GATA3 [28]. Th2 cells express CCR 3 and -4 as surface markers. Effector T cells are the target of several clinically effective therapies. For instance, it was shown in patients with IBD that therapeutic anti-TNF antibodies induce T-cell apoptosis, when co-cultured with CD14+ macrophages [29]. Recently, an additional T-cell pool moved into the focus of IBD pathogenesis. IL-33, a cytokine of the IL-1 family, IL-23 and IL-25 are able to expand so-called innate lymphoid cells (ILCs). While these cells show normal lymphocyte morphology, they lack antigen receptors derived from rearrangement. Different subsets of ILCs have been proposed including NK cells, ROR $\gamma \mathrm{T}$ - dependent ILC and RORa-dependent ILC2 and others. ILCs secrete IL-5, IL-9 and IL-13 and seem to play a role in IBD as well as tissue fibrosis, infection and allergic reaction [30-32].

The role of B cells IBD is only poorly examined and understood. This cell type primarily resides in the lymphoid follicles of the gastrointestinal tract and as plasma cells in the stroma of the lamina propria. IgA and IgM are constantly secreted by intestinal B cells and an important component of the epithelial barrier [33]. In IBD, a reduced expression secretory IgA by dysfunctional B cells can be found [34]. Furthermore, anti-epithelial cell autoantibodies have been proposed in UC, highlighting the potential role of $B$ cells in these patients [35]. It was also shown that peripheral blood B cells constitutively secrete proinflammatory IL-8 in patients with CD. This IL-8 production by peripheral B cells correlated directly with disease severity (fig. 1) [36].

In summary, cells of the innate and the adaptive immune system have been identified as the key regulators of IBD. The innate immune system initiates an exaggerated inflammatory response and cells of the adaptive immune system contribute to the preservation of this pathologic condition. Using new tools, such as next-generation se- 
quencing [37] techniques or new imaging technologies [38], could help to gain an even better understanding of the underlying pathophysiology of CD and UC and contribute to the identification of new therapeutic targets.

\section{Disclosure Statement}

The authors have no conflicts of interest to disclose.

\section{References}

1 Crohn B, Rosenberg H: The sigmoidoscopic 14 Denning TL, Wang Y, Patel SR, Williams IR, picture of chronic ulcerative colitis (non-specific). Am J Med Sci 1925;220-228.

2 Foersch S, Waldner MJ, Neurath MF: Colitis and colorectal cancer. Dig Dis 2012;30:469476.

-3 Schroeder BO, Wu Z, Nuding S, Groscurth S, Marcinowski M, Beisner J, et al: Reduction of disulphide bonds unmasks potent antimicrobial activity of human $\beta$-defensin 1 . Nature 2011;469:419-423.

-4 Günther C, Martini E, Wittkopf N, Amann K, Weigmann B, Neumann H, et al: Caspase- 8 regulates TNF- $\alpha$-induced epithelial necroptosis and terminal ileitis. Nature 2011;477: 335-339.

5 Xavier RJ, Podolsky DK: Unravelling the pathogenesis of inflammatory bowel disease. Nature 2007;448:427-434.

-6 Rakoff-Nahoum S, Hao L, Medzhitov R: Role of toll-like receptors in spontaneous commensal-dependent colitis. Immunity 2006; 25 : 319-329.

7 Nenci A, Becker C, Wullaert A, Gareus R, van Loo G, Danese S, et al: Epithelial NEMO links innate immunity to chronic intestinal inflammation. Nature 2007;446:557-561.

8 Franchimont D: Deficient host-bacteria interactions in inflammatory bowel disease? The toll-like receptor (TLR)-4 Asp299gly polymorphism is associated with Crohn's disease and ulcerative colitis. Gut 2004;53:987992.

-9 Szu-Hee L, Starkey P, Gordon S: Quantitative analysis of total macrophage content in adult mouse tissues. J Exp Med 1985;161:475-489.

10 Bar-on L, Zigmond E, Jung S: Management of gut inflammation through the manipulation of intestinal dendritic cells and macrophages? Semin Immunol 2011;23:58-64.

11 Smythies LE, Sellers M, Clements RH, Mosteller-Barnum M, Meng G, Benjamin WH, et al: Human intestinal macrophages display profound inflammatory anergy despite avid phagocytic and bacteriocidal activity. J Clin Invest 2005;115:66-75.

12 Smythies LE, Shen R, Bimczok D, Novak L, Clements RH, Eckhoff DE, et al: Inflammation anergy in human intestinal macrophages is due to Smad-induced IкBa expression and NF- $\kappa B$ inactivation. J Biol Chem 2010;285: 19593-19604.

13 Kamada N, Hisamatsu T: Unique CD14+ intestinal macrophages contribute to the pathogenesis of Crohn disease via IL-23/IFN- $\gamma$ axis. J Clin Invest 2008;118:2269-2280.
Pulendran B: Lamina propria macrophages and dendritic cells differentially induce regulatory and interleukin-17-producing T-cell responses. Nat Immunol 2007;8:1086-1094.

15 Kamada N, Hisamatsu T: Abnormally differentiated subsets of intestinal macrophage play a key role in Th1-dominant chronic colitis through excess production of IL-12 and IL-23 in response to bacteria. J Immunol 2005; 175 : 6900-6908.

16 Rimoldi M, Chieppa M, Salucci V, Avogadri F, Sonzogni A, Sampietro GM, et al: Intestinal immune homeostasis is regulated by the crosstalk between epithelial cells and dendritic cells. Nat Immunol 2005;6:507-514.

17 Rescigno M, Di Sabatino A: Dendritic cells in intestinal homeostasis and disease. J Clin Invest 2009;119:2441-2450.

18 Chieppa M, Rescigno M, Huang AYC, Germain RN: Dynamic imaging of dendritic cell extension into the small bowel lumen in response to epithelial cell TLR engagement. J Exp Med 2006;203:2841-2852.

19 Lathrop SK, Santacruz NA, Pham D, Luo J, Hsieh C-S: Antigen-specific peripheral shaping of the natural regulatory T-cell population. J Exp Med 2008;205:3105-1317.

20 Powrie F, Leach MW, Mauze S, Menon S, Caddle LB, Coffman RL: Inhibition of Th1 responses prevents inflammatory bowel disease in scid mice reconstituted with CD45RBhi CD4+ T cells. Immunity 1994;1:553-562.

21 Zhou L, Littman DR: Transcriptional regulatory networks in Th17 cell differentiation. Curr Opin Immunol 2009;21:146-152.

22 Murphy K, Ouyang W: Signaling and transcription in T-helper development. Annu Rev Immunol 2000;451-494.

23 Kamiya S, Owaki T, Morishima N, Fukai F, Mizuguchi J, Yoshimoto T: An indispensable role for STAT1 in IL-27-induced T-bet expression but not proliferation of naive $\mathrm{CD} 4+$ T cells. J Immunol 2004;173:3871-3877.

24 Takeda A, Hamano S, Yamanaka A, Hanada T, Ishibashi T, Mak TW, et al: Cutting edge: role of IL-27/WSX-1 signaling for induction of T-bet through activation of STAT1 during initial Th1 commitment. J Immunol 2003; 170:4886-4890.

25 Schraml BU, Hildner K, Ise W, Lee WL, Smith WA, Solomon B, et al: The AP-1 transcription factor Batf controls $\mathrm{T}(\mathrm{H}) 17$ differentiation. Nature 2009;460:405-409.
Neurath M: IL-23: a master regulator in Crohn disease. Nat Med 2007;13:26-28.

27 Leppkes M, Becker C, Ivanov II, Hirth S, Wirtz S, Neufert C, et al: ROR $\gamma$-expressing Th17 cells induce murine chronic intestinal inflammation via redundant effects of IL-17A and IL-17F. Gastroenterology 2009;136:257267.

28 Ho I-C, Tai T-S, Pai S-Y: GATA3 and the Tcell lineage: essential functions before and after T-helper-2 cell differentiation. Nat Rev Immunol 2009;9:125-135.

29 Atreya R, Zimmer M, Bartsch B, Waldner MJ, Atreya I, Neumann $\mathrm{H}$, et al: Antibodies against tumor necrosis factor (TNF) induce T-cell apoptosis in patients with inflammatory bowel diseases via TNF receptor 2 and intestinal CD14+ macrophages. Gastroenterology 2011;141:2026-2038.

-30 Scanlon ST, McKenzie ANJ: Type 2 innate lymphoid cells: new players in asthma and allergy. Curr Opin Immunol 2012;24:707-712.

31 Sonnenberg GF, Monticelli LA, Alenghat T, Fung TC, Hutnick NA, Kunisawa J, et al: Innate lymphoid cells promote anatomical containment of lymphoid-resident commensal bacteria. Science 2012;336:1321-1325.

- 32 Sawa S, Lochner M, Satoh-Takayama N, Dulauroy $S$, Bérard $M$, Kleinschek $M$, et al: ROR $\gamma \mathrm{t}+$ innate lymphoid cells regulate intestinal homeostasis by integrating negative signals from the symbiotic microbiota. Nat Immunol 2011;12:320-326.

33 Brandtzaeg P: Induction of secretory immunity and memory at mucosal surfaces. Vaccine 2007;25:5467-5484.

34 Brandtzaeg P, Carlsen HS, Halstensen TS: The B-cell system in inflammatory bowel disease. Adv Exp Med Biol 2006;579:149-167.

35 Goetz M, Atreya R, Ghalibafian M, Galle PR, Neurath MF: Exacerbation of ulcerative colitis after rituximab salvage therapy. Inflamm Bowel Dis 2007;13:1365-1368.

36 Noronha A, Liang Y: Hyperactivated B cells in human inflammatory bowel disease. J Leukoc Biol 2009;86:1007-1016.

37 Jostins L, Ripke S, Weersma RK, Duerr RH, McGovern DP, Hui KY, et al: Host-microbe interactions have shaped the genetic architecture of inflammatory bowel disease. Nature 2012;491:119-124.

38 Schürmann S, Foersch S, Atreya R, Neumann $\mathrm{H}$, Friedrich O, Neurath MF, et al: Label-free imaging of inflammatory bowel disease using multiphoton microscopy Gastroenterology 2013, E-pub ahead of print. 